\title{
The optimal dimensional design of machining technologies
}

\author{
Alexei Toca*, Aurel Stroncea, Ion Stîngaci and Ion Ruşica \\ Technical University of Moldova, Department of Machines Manufacturing Technology, str. \\ Studentilor, 9, Chisinau, Republic of Moldova
}

\begin{abstract}
The paper discusses the design methodology of optimal technological processes of machining, bearing in mind that these processes are related to discrete processes. Discreteness is manifested at different levels: the process consists of a certain number of operations, the operation consists of a number of adjustments and the adjustment consists of a number of operation elements. Each of these levels is a dimensional object with its own internal dimensional structure. The links between dimensional levels are complex and the process is iterative with the ability to return to a higher level.
\end{abstract}

\section{Introduction}

The existing classical methods to design technological process are complex and do not provide the required level of quality and reliability of technologies. The theory of manufacturing engineering to design the technology has a well-developed content part, but it is not sufficiently formalized. The designing processes for complex parts and for conditions of use of the $\mathrm{CNC}$ machine tools is more challenging in terms of both general approach and from the point of view of formalizing the process used and logicalmathematical apparatus. The design process of the technologies should be considered more perfect if it requires a smaller number of information changes, including a smaller number of iterations. The technological process should be considered more perfect when it requires for use a smaller number of transformations of matter and energy which are also displayed via relevant information. One of the most complicated problems during the elaboration of the manufacturing process is the synthesis of dimensional structure. Not only dimensional analysis of a developed manufacturing process but also to achieve on this basis the optimum dimensional structure it is necessary to execute. The machining processes are referred to discrete processes. The discreteness is manifested at different levels: the process consists of a certain number of operations, the operation consists of a number of adjustments and the adjustment consists of a number of operation elements. Each of these levels is a dimensional object with its own internal dimensional structure. The links between dimensional levels are complex and the optimization process is iterative with the ability to return to a higher level.

\footnotetext{
*Corresponding author: a toca@mail.utm.md
} 
The dimensional analysis takes a special place during designing technology, concerning the stage of designing and also the stage of technological designing [1-6]. The purpose of this process is to establish the values of working dimensions and working tolerances for the part at each technological element, so that the cost of machining would be minimally possible.

\section{Optimality of the machining technologies}

Machining technologies represent sophisticated multilevel structures and for this reason achieving of the optimality is required for each structural level: machining technology, technological operation, technological mounting, technological element. The modern technologies are based on the use of CNC machine-tools and machining centers so the dimensional and information discontinuities inherent in multi-operational technologies are gradually disappearing. In these conditions the main level for optimization is technological operation and technological mounting. One of the criteria of technological operation's optimality has a structural nature. The manufacturing operation are considered optimum, if in the technological dimensional chain's structures of all technological dimensional chains, the number of the technological sizes is minimum [3]. This condition is respected if each constructive size is represented by a single technological size in the technological dimensional chain, and in dimensional chains for machining allowances, each machining allowance is determined by two technological sizes or one technological size and one size on blank. The minimum possible number of the technological sizes $N_{T_{M I N}}$ is determined from the relation $N_{T_{M I N}}=N_{C}+2 \cdot N_{A d}$, where $N_{C}$ - number of the constructive sizes, $N_{A d}$ - number of machining allowances.

It is necessary to note, that the dimensional optimality is not entirely characterized by the number $N_{T}$, but it is necessary to take into account the growth (sometimes complicated) of the technological sizes accuracy (a case of formation of the sizes as closing link of dimensional chains).

Other criteria for optimization are minimum of the sizes and minimum of the tolerances of the machining allowances, such that the use of low-energy and time consumption. In the case of big values of the machining allowances or of big values of tolerances of machining allowances, the increase of the number of the technological elements is possible. This means that the optimal solution is brought on another level.

The evaluation of the conditions of size's accuracy formation depending on structures of dimensional constructive and technological links represents interest. There were analyzed examples of details machining with different constructive dimensional links with a formation of the sizes from technological locating datum surface and from adjustment surfaces.

One of the variants is demonstrated on Figure 1. The all constructive sizes are given from constructive base - surface 7 and the technological sizes are given also from locating datum surface 7 .

In a ratio of tolerances $\omega$ for the technological elements $i=1,2$ it is possible to write: $\omega_{A_{t}^{i}}=\omega_{A_{C}^{i}}, \omega_{B_{t}^{i}}=\omega_{B_{c}^{i}}, \omega_{C_{t}^{i}}=\omega_{C_{c}^{i}}, \omega_{E_{t}^{i}}=\omega_{E_{c}^{1}}$.

From these expressions follows that the accuracy of the technological dimensions must be equal to the accuracy of the corresponding constructive dimensions. 

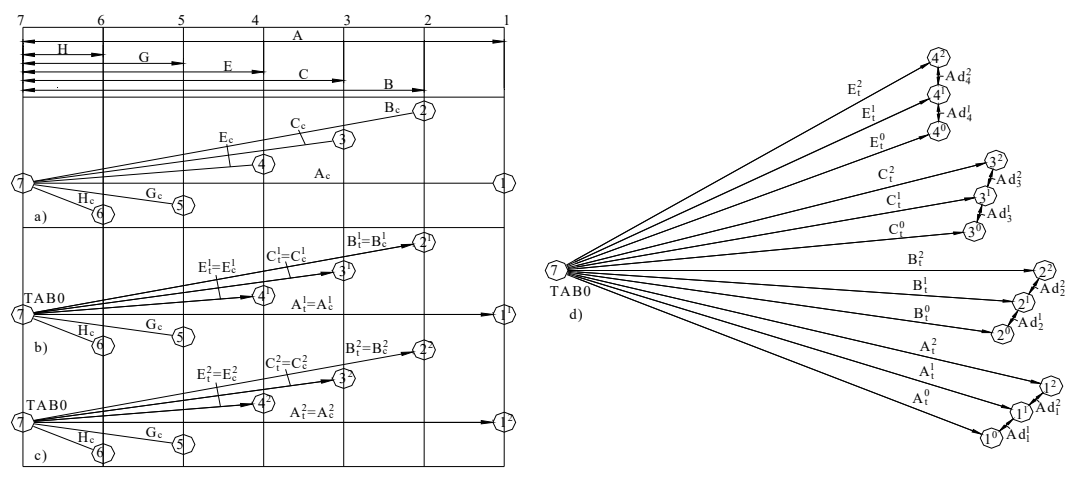

Fig. 1. Example 1 of constructive variant of a detail and the appropriate structures of the dimensional technological links.

Resolving the dimension chains for the machining allowances taking into account the effect of error's compensation [7] one can write down:

$$
\begin{aligned}
& \omega_{A d_{1}^{1}}=\left(\omega_{A_{t}^{0}}+\omega_{A_{t}^{1}}\right)-2 \omega_{1^{0} 1^{1}}^{c o m p} \\
& \omega_{A d_{1}^{2}}=\left(\omega_{A_{t}^{1}}+\omega_{A_{t}^{2}}\right)-2 \omega_{1_{1} 1^{2}}^{c o m p} \\
& \omega_{A d_{2}^{1}}=\left(\omega_{B_{t}^{0}}+\omega_{B_{t}^{1}}\right)-2 \omega_{2^{0} 2^{1}}^{c o m p} \\
& \omega_{A d_{2}^{2}}=\left(\omega_{B_{t}^{1}}+\omega_{B_{t}^{2}}\right)-2 \omega_{2^{1} 2^{2}}^{\text {comp }} \\
& \omega_{A d_{3}^{1}}=\left(\omega_{C_{t}^{0}}+\omega_{C_{t}^{1}}\right)-2 \omega_{3^{0} 3^{1}}^{c o m p} \\
& \omega_{A d_{3}^{2}}=\left(\omega_{C_{t}^{1}}+\omega_{C_{t}^{2}}\right)-2 \omega_{3^{1} 3^{2}}^{c c o m p} \\
& \omega_{A d_{4}^{1}}=\left(\omega_{E_{t}^{0}}+\omega_{E_{t}^{1}}\right)-2 \omega_{4^{0} 4^{1}}^{c o m p} \\
& \omega_{A d_{4}^{2}}=\left(\omega_{E_{t}^{1}}+\omega_{E_{t}^{2}}\right)-2 \omega_{4_{4}^{1} 4^{2}}^{c o m p}
\end{aligned}
$$

From (1-8) follows, that the tolerance of a machining allowances deleted with a surface is defined by an accuracy of the appropriate technological sizes at two sequential stages of machining and the accuracy of sizes between technological locating datum surface and adjustment surface at two sequential stages of machining. Another variant is demonstrated on Figure 2. Constructive dimensions are given from the surface 1, however the technological dimensions are given from locating datum surface 7 . In a ratio of tolerances $\omega$ for the technological elements $i=1,2$ it is possible to write: $\omega_{A_{t}^{i}} \leq \omega_{A_{C}^{i}}$, $\omega_{B_{t}^{i}}+\omega_{A_{t}^{i}} \leq \omega_{B_{C}^{i}}, \omega_{C_{t}^{i}}+\omega_{A_{t}^{i}} \leq \omega_{C_{c}^{i}}, \omega_{E_{t}^{i}}+\omega_{A_{t}^{i}} \leq \omega_{E_{C}^{1}}$. From these expressions follows that the accuracy of the technological sizes must be higher than the accuracy of the corresponding 
constructive sizes. For the case $A_{c}=100(I T 13)$ and $B_{c} / A_{c}=0.02 \ldots 0.98$ the number of tolerance units of the technological size $a_{B t}$ is within $0.13 \ldots 0.8$ of the number of constructive size's tolerance units (figure 3). The accuracy of the technological size increases from IT13 to IT9...IT12. This technological scheme for small sizes is especially disadvantageous.
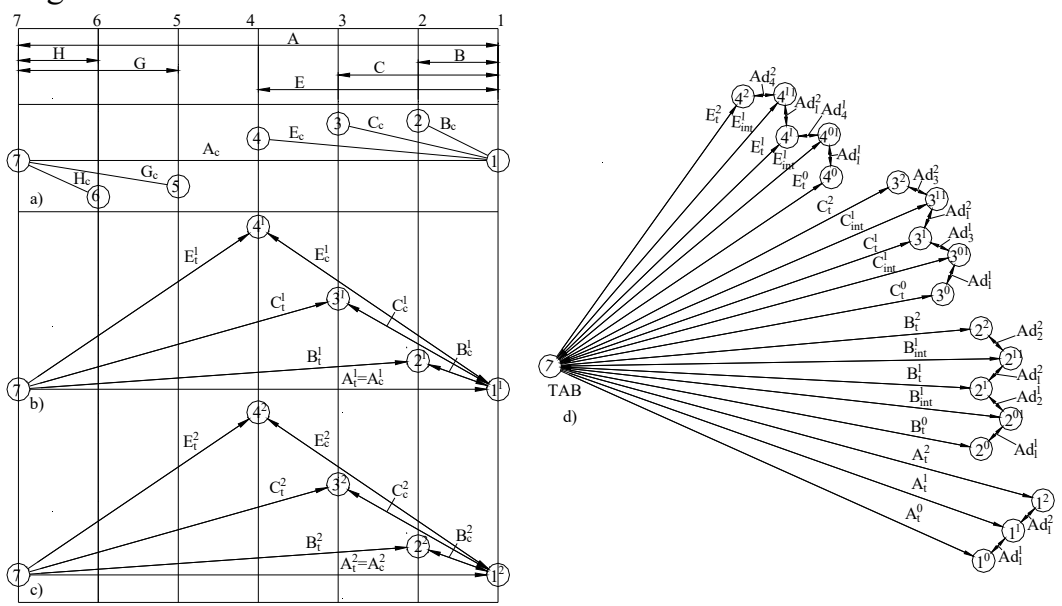

Fig. 2. Example 2 of constructive variant of a detail and the appropriate structures of the dimensional technological links.
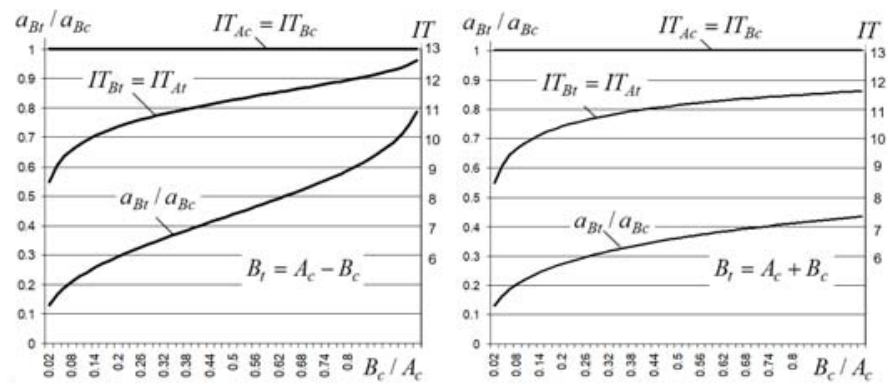

Fig. 3. The accuracy's increase of the technological sizes in comparison with the accuracy of the constructive sizes.

Resolving the dimension chains for the machining allowances taking into account the effect of error's compensation one can write down:

$$
\begin{aligned}
& \omega_{A d_{1}^{1}}=\left(\omega_{A_{t}^{0}}+\omega_{A_{t}^{1}}\right)-2 \omega_{1^{0} 1^{1}}^{\text {comp }} \\
& \omega_{A d_{1}^{2}}=\left(\omega_{A_{t}^{1}}+\omega_{A_{t}^{2}}\right)-2 \omega_{1^{1} 1^{2}}^{c o m p} \\
& \omega_{A d_{2}^{1}}=\left(\omega_{B_{t}^{0}}+\omega_{B_{t}^{1}}\right)-2 \omega_{2^{0} 2^{1}}^{\text {comp }}+\left(\omega_{A_{t}^{0}}+\omega_{A_{t}^{1}}\right)-2 \omega_{1^{0} 1}^{c o m p} \\
& \omega_{A d_{2}^{2}}=\left(\omega_{B_{t}^{1}}+\omega_{B_{t}^{2}}\right)-2 \omega_{2^{1} 2^{2}}^{c o m p}+\left(\omega_{A_{t}^{1}}+\omega_{A_{t}^{2}}\right)-2 \omega_{1_{1}^{1}}^{\text {comp }}
\end{aligned}
$$




$$
\begin{aligned}
& \omega_{A d_{3}^{1}}=\left(\omega_{C_{t}^{0}}+\omega_{C_{t}^{1}}\right)-2 \omega_{3^{3} 3^{1}}^{c o m p}+\left(\omega_{A_{t}^{0}}+\omega_{A_{t}^{1}}\right)-2 \omega_{1^{0} 1^{1}}^{c o m p} \\
& \omega_{A d_{3}^{2}}=\left(\omega_{C_{t}^{1}}+\omega_{C_{t}^{2}}\right)-2 \omega_{3^{3} 3^{2}}^{c o m p}+\left(\omega_{A_{t}^{1}}+\omega_{A_{t}^{2}}\right)-2 \omega_{1_{1}{ }^{2}}^{c o m p} \\
& \omega_{A d_{4}^{1}}=\left(\omega_{E_{t}^{0}}+\omega_{E_{t}^{1}}\right)-2 \omega_{4_{4}^{0}{ }^{1}}^{c o m p}+\left(\omega_{A_{t}^{0}}+\omega_{A_{t}^{1}}\right)-2 \omega_{1_{1} 1^{1}}^{c o m p} \\
& \omega_{A d_{4}^{2}}=\left(\omega_{E_{t}^{1}}+\omega_{E_{t}^{2}}\right)-2 \omega_{4^{1} 4^{2}}^{c o m p}+\left(\omega_{A_{t}^{1}}+\omega_{A_{t}^{2}}\right)-2 \omega_{1_{1} 1^{2}}^{c o m p}
\end{aligned}
$$
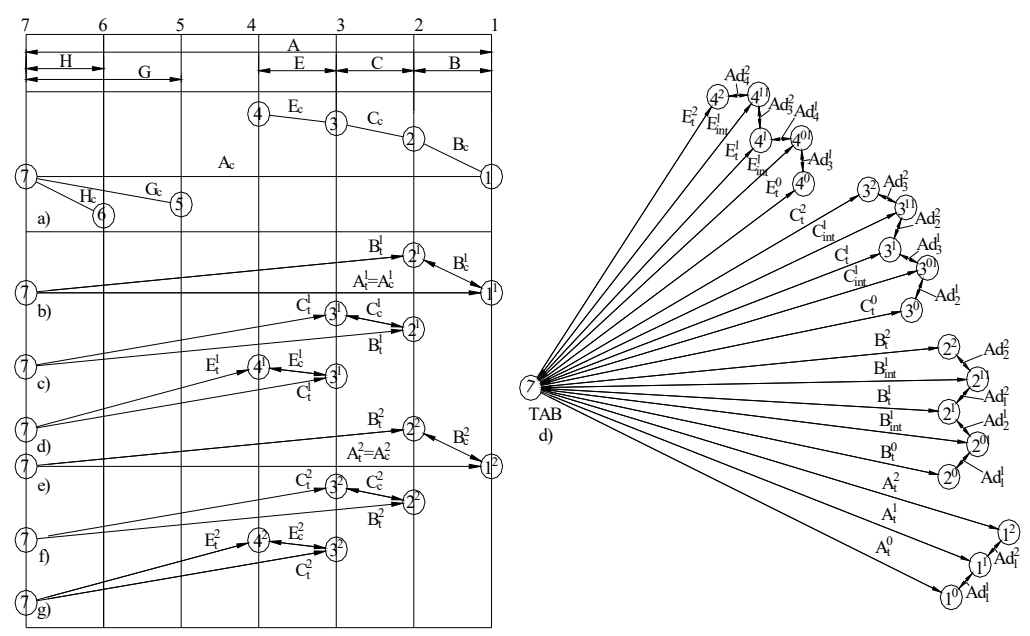

Fig. 4. Example 3 of constructive variant of a detail and the appropriate structures of the dimensional technological links.

From (9-16) follows, that the tolerance of a machining allowances deleted with a surface is defined by an accuracy of the appropriate technological sizes at two sequential stages of machining and the accuracy of sizes between technological locating datum surface and adjustment surface at two sequential stages of machining. It is possible to draw an output, that in case of use at machining instead of technological locating datum surface the adjustment surface, thus the tolerance of the machining allowances depends on tolerances of all technological sizes at two adjacent stages of machining, defining a position of a processed surface concerning each adjustment surfaces used till technological locating datum surface. Another variant is demonstrated on Figure 4. Constructive dimensions are given in chain from the surface 1 , however the technological dimensions are given from locating datum surface 7. In a ratio of tolerances $\omega$ for the technological elements $i=1,2$ it is possible to write: $\omega_{A_{t}^{i}} \leq \omega_{A_{c}^{i}}, \omega_{B_{t}^{i}}+\omega_{A_{t}^{i}} \leq \omega_{B_{c}^{i}}, \omega_{C_{t}^{i}}+\omega_{B_{t}^{i}} \leq \omega_{C_{c}^{i}}, \omega_{E_{t}^{i}}+\omega_{C_{t}^{i}} \leq \omega_{E_{c}^{1}}$. From these expressions follow, so as in the previous case, that the accuracy of the technological sizes is higher than the accuracy of the constructive sizes (Figure 3). Resolving the dimension chains for the machining allowances taking into account the effect of error's compensation one can write down:

$$
\omega_{A d_{1}^{1}}=\left(\omega_{A_{t}^{0}}+\omega_{A_{t}^{1}}\right)-2 \omega_{1_{1}^{1}}^{c o m p}
$$




$$
\begin{aligned}
& \omega_{A d_{1}^{2}}=\left(\omega_{A_{t}^{1}}+\omega_{A_{t}^{2}}\right)-2 \omega_{1^{1} 1^{2}}^{\text {comp }} \\
& \omega_{A d_{2}^{1}}=\left(\omega_{B_{t}^{0}}+\omega_{B_{t}^{1}}\right)-2 \omega_{2^{0} 2^{1}}^{c o m p}+\left(\omega_{A_{t}^{0}}+\omega_{A_{t}^{1}}\right)-2 \omega_{1^{0} 1^{1}}^{c o m p} \\
& \omega_{A d_{2}^{2}}=\left(\omega_{B_{t}^{1}}+\omega_{B_{t}^{2}}\right)-2 \omega_{2^{1} 2^{2}}^{c o m p}+\left(\omega_{A_{t}^{1}}+\omega_{A_{t}^{2}}\right)-2 \omega_{1_{1} 1^{2}}^{\text {comp }} \\
& \omega_{A d_{3}^{1}}=\left(\omega_{C_{t}^{0}}+\omega_{C_{t}^{1}}\right)-2 \omega_{3^{0} 3^{1}}^{c o m p}+\left(\omega_{B_{t}^{0}}+\omega_{B_{t}^{1}}\right)-2 \omega_{2^{0} 2^{1}}^{\text {comp }} \\
& \omega_{A d_{3}^{2}}=\left(\omega_{C_{t}^{1}}+\omega_{C_{t}^{2}}\right)-2 \omega_{3^{1} 3^{2}}^{c o m p}+\left(\omega_{B_{t}^{1}}+\omega_{B_{t}^{2}}\right)-2 \omega_{2_{1} 2^{2}}^{\text {comp }} \\
& \omega_{A d_{4}^{1}}=\left(\omega_{E_{t}^{0}}+\omega_{E_{t}^{1}}\right)-2 \omega_{4^{0} 4^{1}}^{\text {comp }}+\left(\omega_{C_{t}^{0}}+\omega_{C_{t}^{1}}\right)-2 \omega_{3^{0} 3^{1}}^{\text {comp }} \\
& \omega_{A d_{4}^{2}}=\left(\omega_{E_{t}^{1}}+\omega_{E_{t}^{2}}\right)-2 \omega_{4^{1} 4^{2}}^{\text {comp }}+\left(\omega_{C_{t}^{1}}+\omega_{C_{t}^{2}}\right)-2 \omega_{3^{1} 3^{2}}^{\text {comp }}
\end{aligned}
$$

The difference lies in the fact that the machining allowance's tolerances is influenced by the tolerances of the technological sizes of the neighbors in a chain.

\section{Conclusions}

The schemes of machining with a formation of sizes from technological locating datum surface are characterized by unfavorable conditions of accuracy formation for constructive sizes. In some cases, the machining accuracy can be significantly greater than the accuracy of constructive sizes. The reason for this phenomenon is that the constructive dimensional structure of the part does not coincide with the dimensional structure of the technological system. From the point of view of the accuracy assurance strategy, the optimality of the technological process is ensured by the formation of each constructive size by its corresponding technological size. The machining allowances may have sufficiently large tolerances because their tolerance depends on the multiples change of the technological bases, and the entire size chain to the technological base. High tolerances for the machining allowances require the establishing the large cutting depths which sometimes require an additional operational elements.

\section{References}

1. M. Thilak, N. Senthil Kumar, Int. J. Appl. Eng. Research, 10, 78 (2015)

2. F. González Contreras, Int. J. Adv. Man. Techn., 62, 9 - 12 (2012)

3. A.V. Perminov, The News of High Schools. Mechanical Engineering, 4, 26-30 (2002)

4. Y.A. Mokrushin, V.Y. Shamin, Univ. proc. Volga region. Machine sciences and building, 1, 123-130 (2013)

5. E.G. Rahmilevich, Tehnologiâ Mašinostroeniâ, 1, 53-56 (2013)

6. W. Li, G. Bai, C. Zhang and B. Wang, Int. J. Prod Research, 38, 6 (2000)

7. A. Toca, I. Stingaci, I. Rushica, Proceedings of $16^{\text {th }}$ International Conference ModTech, (ModTech Publishing House, 953-956, 2012) 